\title{
ChemComm
}

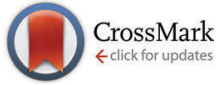

Cite this: Chem. Commun., 2014 50, 12389

Received 15th August 2014 Accepted 27th August 2014

DOI: $10.1039 / \mathrm{c} 4 \mathrm{cc} 06438 \mathrm{k}$

www.rsc.org/chemcomm

\section{Enhanced Raman and luminescence spectra from co-encapsulated silicon quantum dots and $\mathrm{Au}-\mathrm{Ag}$ nanoalloys $\dagger$}

\author{
Noor Aniza Harun, ${ }^{\text {ab }}$ Benjamin R. Horrocks ${ }^{\star^{a}}$ and David A. Fulton ${ }^{\star a}$
}

We report an approach to enhance simultaneously luminescence and SERS signals with a single excitation wavelength by co-encapsulating silicon quantum dots and $\mathrm{Au}-\mathrm{Ag}$ alloy nanoparticles encoded with Raman reporter molecules inside polymeric nanoparticles. The SERSluminescence enhancement exploits the large Stokes shift of silicon quantum dots, which allows 'room' for the display of a Raman spectrum.

Silicon quantum dots (SiQDs) $)^{1}$ are nanometre-sized crystals of silicon which are of interest on account of their size-dependent luminescence characteristics. A characteristic of special interest is the presence of a large Stokes shift which arises as a result of the combination of direct and indirect gaps in bulk $\mathrm{Si}^{2}$ This factor raises the intriguing possibility that further spectral information can be encoded in the spectral region between the absorbance and luminescence of the SiQDs. In particular, the possibility to encode a Raman spectrum would be appealing as it may lead to dual luminescence and SERSencoded particles with increased utility as probes in biological systems $^{3}$ and as components in anti-counterfeiting technologies.

Dual luminescence and SERS-encoded particles may be prepared by co-encapsulating both SiQDs and a SERS reporter molecule within the polymeric matrix of a polymer nanoparticle. Since Raman spectra are inherently weak and the overall brightness of SiQDs can be restricted, ${ }^{4}$ to ensure sufficiently high spectral intensities to be useful in applications it is necessary to enhance both Raman and luminescence with the aid of noble metals such as $\mathrm{Ag}$ or $\mathrm{Au}$. We recently demonstrated ${ }^{5}$ that the co-encapsulation of SiQDs and Au nanoparticles (NPs) resulted in composite NPs possessing metal-enhanced luminescence properties. Here, we report the development of multi-component polymer composite nanoparticles composed of SiQDs and Ag-Au alloy NPs encoded with Raman reporter molecules which are co-encapsulated inside polymeric NPs.

\footnotetext{
${ }^{a}$ Chemical Nanoscience Laboratory, School of Chemistry, Newcastle University,

Newcastle upon Tyne, NE1 7RU, UK. E-mail: b.r.horrocks@ncl.ac.uk, d.a.fulton@ncl.ac.uk

${ }^{b}$ School of Fundamental Science, Universiti Malaysia Terengganu, 21030, Kuala Terengganu, Terengganu, Malaysia

$\dagger$ Electronic supplementary information (ESI) available: All synthetic procedures and additional characterization. See DOI: 10.1039/c4cc06438k
}

These composite NPs offer dual optical characteristics associated with SERS of the Raman-active Ag-Au alloy NPs and metal-enhanced luminescence effects associated with SiQDs. Preliminary studies investigating their SERS and luminescence enhancement effects reveal simultaneous enhancements of the luminescence of SiQDs ( $\sim 17$ fold) and SERS (up to five-fold).

Our composite NPs were prepared by co-encapsulating luminescent SiQDs and a SERS reporter molecule within a nano-sized polymer matrix consisting of poly(divinylbenzene). 2-Naphthalenethiol was chosen as the SERS-active molecule on account of its strong sharp Raman signals, and its thiol group allows its conjugation to the surface of Ag-Au alloy $\mathrm{NPs}^{6}$ ( $30 \mathrm{~nm} \pm 3.9 \mathrm{~nm}$ diameter) to enhance the SERS signal. To further aid the dispersion of the SERS-labelled $\mathrm{Ag}-\mathrm{Au}$ alloy NPs in organic media, linear thiol-terminated polystyrene chains were also conjugated onto their surfaces utilizing a previously reported method. ${ }^{7}$ The resulting polymer coated SERS-labelled Ag-Au alloy NPs (PS-SERS-NPs) and SiQDs were co-encapsulated inside a polymer matrix utilizing mini-emulsion procedures ${ }^{7,8}$ (see ESI $\dagger$ ) to afford composite polymeric NPs of $200 \mathrm{~nm}-230 \mathrm{~nm}$ in diameter with reasonably narrow particle size distributions as determined by dynamic light scattering (DLS). A control composite NP absent in SiQDs was also prepared. The various species utilized in this work are summarized in Scheme 1.

The size and morphology of the polymer composite NPs was investigated by TEM (Fig. 1), revealing their average diameter to be approximately $100-200 \mathrm{~nm}$, in good agreement with results obtained from DLS measurements. The encapsulated PS-SERS-NPs are clearly evident as visible spheres of high TEM contrast (black), distributed evenly within the lower contrast poly(divinylbenzene) matrix(grey). Furthermore, the existence of un-encapsulated SERS-labelled Au-Ag NPs and "empty" polymer NPs was also observed. These two features have been prevalent in previous reports of polymer composite NPs formed by miniemulsion polymerization ${ }^{9}$ and we anticipate that these issues could be addressed through further development of the encapsulation procedures. Because of the low scattering factor of SiQDs, TEM cannot confirm the existence or absence of SiQDs within the polymer NPs. 
<smiles>Sc1ccc2ccccc2c1</smiles>

2-napthalene thiol

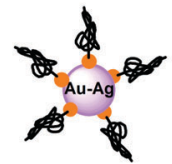

PS-SERS-NPS
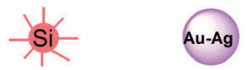
SiQDs

$\mathrm{Ag}-\mathrm{Au}$ alloy-NPs

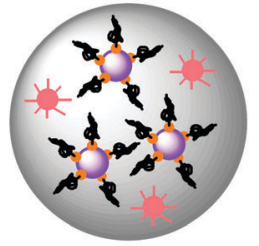

Composite NPs

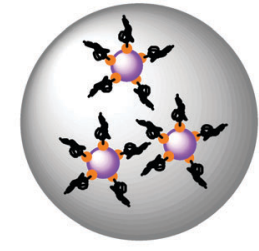

Composite NPs absent in SiQDs
Scheme 1 Structure of the SERS reporter molecule 2-napthlene thiol and cartoon representations of the various NPs used throughout this study.

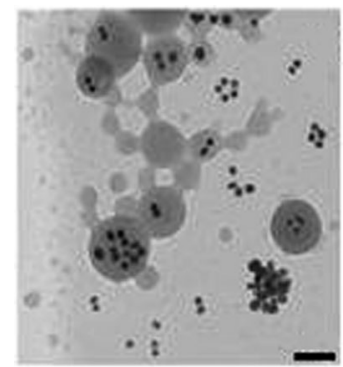

Fig. 1 PS-SERS-NPs (92000× magnification). Because of the low scattering factor of $\mathrm{Si}$, TEM cannot confirm the presence or absence of SiQDs within the polymer matrix. Scale bar $=100 \mathrm{~nm}$.

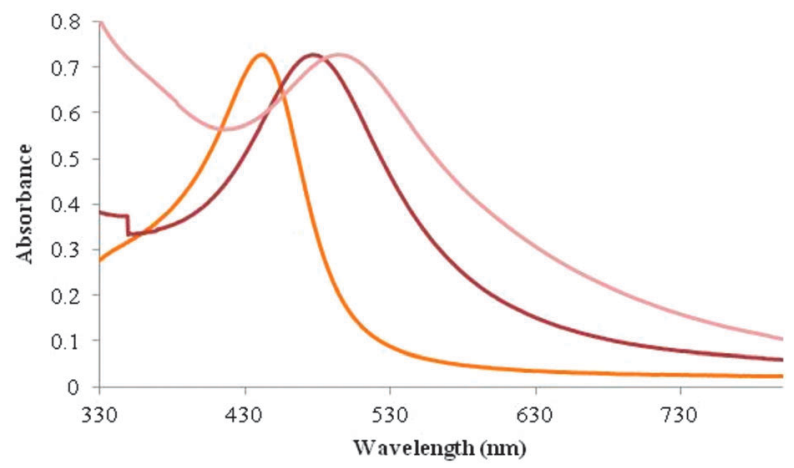

Fig. 2 Comparison of the UV-Vis spectra of citrate-stabilised aqueous $\mathrm{Ag}-\mathrm{Au}$ alloy NPs dispersed in water (orange-coloured trace), PS-SERSNPs dispersed in THF (dark red-coloured trace) and the composite NPs dispersed in THF (pink-coloured trace).

The UV-Vis spectra (Fig. 2) indicates the surface plasmon resonance (SPR) band of the composite NPs (498 nm) is red-shifted relative to the SPR band of the un-encapsulated PS-SERS-NPs $(480 \mathrm{~nm})$ in THF. Both of these species are significantly red-shifted relative to the citrate-stabilized Ag-Au alloy NPs (448 nm) dispersed in water. This red shifting is expected on account of the different refractive indices of the surrounding media of the metal NPs. ${ }^{8}$
As reported previously, ${ }^{5}$ the red shifting of the SPR of the composite NPs is also expected as a consequence of small changes in dielectric environments and the capping layer surrounding the polymer functionalized $\mathrm{Ag}-\mathrm{Au}$ alloy NPs. It is well-known ${ }^{10}$ that the increase in the refractive index of the surrounding medium and the decrease in the inter-particle distance between neighbouring metal NPs influences the spectral features of samples containing dispersed metal nanoparticles. The increase in absorption observed at short wavelengths for the composite polymer NPs arises because of absorption from the encapsulated SiQDs, as samples absent in SiQDs do not display this increase. Taken together, these UV-Vis spectroscopic and TEM studies suggest successful encapsulation of the polymer functionalized $\mathrm{Ag}-\mathrm{Au}$ alloy NPs within a polymer matrix.

Raman and luminescence spectra of the composite NPs were investigated by confocal microspectroscopy. The confocal luminescence image obtained (ESI, $\dagger$ S1) from a sample drop-cast onto a glass cover slip reveals bright regions which confirms the existence of SiQDs which are encapsulated inside and/or associated onto the surface of polymer nanoparticles. On the contrary, no luminescence was monitored for the control composite NP absent in SiQDs (data not shown).

To evaluate the SERS and luminescence enhancement behaviour of the composite NPs, the average luminescence/Raman spectra were obtained by averaging the luminescence confocal images over the regions where particles were observed; this process normalises for differences in particle density in the image region (Fig. 3). The obvious changes of SERS in comparison to the conventional Raman spectrum of 2-naphthalenethiol can be observed in the differences in relative intensities of the most intense bands, and the simplification of the SERS spectrum where several bands are either not detected or exhibit a very low intensity. ${ }^{10}$ When we attempted to account for the difference in the numbers of naphthalenethiol molecules in the two spectra of Fig. 3a, we obtained a SERS enhancement of order $10^{3}$. However, these estimates are subject to large uncertainties and may give a misleading impression of the intensity actually measured in the composite NPs. Throughout the paper we therefore report SERS
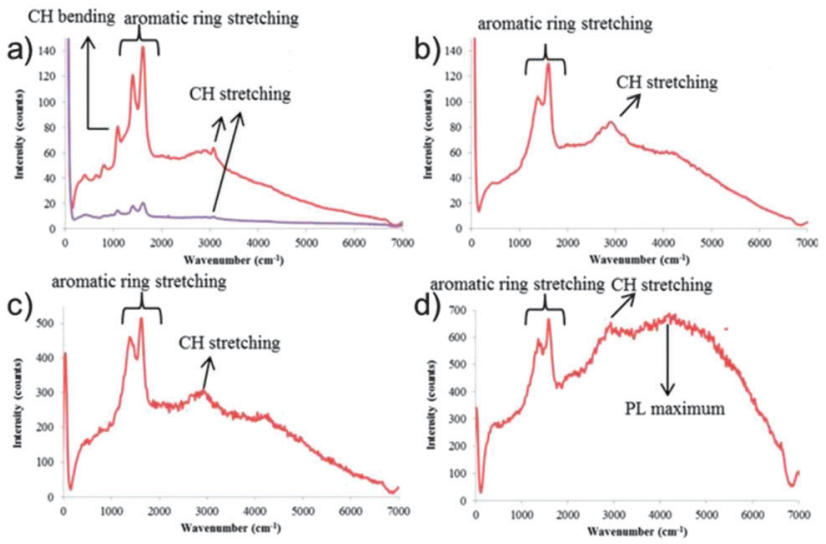

Fig. 3 Average luminescence/Raman spectra of (a) SERS-labelled Ag-Au NPs (red trace) and a film of 2-naphthalenethiol (purple trace) (b) PS-SERS-NPs (c) composite NPs absent in SiQDs and (d) composite NPs. The higher intensity peak at $0 \mathrm{~cm}^{-1}$ is the elastically scattered laser light. The excitation wavelength, $\lambda=488 \mathrm{~nm}$ and the spectrograph employed a grating with 150 lines per $\mathrm{mm}$. 
enhancements as the ratio of intensities between two spectra without attempting to account for the differences in the numbers of 2-napthalenethiol molecules in each spectrum. This gives an enhancement of about seven-fold from Fig. 3a upon comparing a film of 2-napthalenethiol to unencapsulated NPs and about five-fold for encapsulated NPs. Luminescence enhancements are reported similarly by taking the ratio of intensities of two spectra \{composite $\mathrm{NPs}\}:\{$ composite NPs absent in Au-Ag NPs $\}$. The intensities were obtained by integrating each spectrum from $1900 \mathrm{~cm}^{-1}$ to $7000 \mathrm{~cm}^{-1}$ to avoid the main Raman peaks.

The mean luminescence/Raman spectra of un-encapsulated SERSlabelled $\mathrm{Ag}-\mathrm{Au}$ NPs (absent in polystyrene coating) (Fig. 3a) shows significant SERS peaks at $1392-1578 \mathrm{~cm}^{-1}$ which are attributed to the aromatic ring stretching mode of the 2-naphthalenethiol unit. A SERS enhancement up to seven times higher was observed for these SERS-labelled Ag-Au alloy NPs in comparison to the intensity of the conventional Raman spectra of a film of 2-naphthalenethiol. Additionally, small signals at $1069 \mathrm{~cm}^{-1}, 773 \mathrm{~cm}^{-1}$ and $356 \mathrm{~cm}^{-1}$ were also observed which correspond to the $\mathrm{C}-\mathrm{H}$ bending, $\mathrm{C}-\mathrm{H}$ wagging and ring deformation respectively. A weak and small SERS signal at $3059 \mathrm{~cm}^{-1}$ refers to the $\mathrm{C}-\mathrm{H}$ stretching of 2-naphthalenethiol. It should be noted that the observed SERS spectra may be quite different from the normal Raman scattering spectra of the same molecule due to factors such as light polarization at the metal surface, orientation of the chemisorbed species, and other factors such as photo-dissociation or photochemistry at the metal interface. ${ }^{11}$

These major SERS bands were also observed for the PS-SERS-NPs (Fig. 3b). The fact that the SERS effect can be observed for these species is expected because surface modification with linear polystyrene chains results in less dense packing of polymer chains onto the $\mathrm{Au}-\mathrm{Ag}$ NPs surfaces, allowing sufficient space to bind SERS reporter molecules. ${ }^{8}$ However, minor differences in the spectra were observed, including the disappearance of small peaks around $300-1100 \mathrm{~cm}^{-1}$ and the broadening of the peak at $\sim 3000 \mathrm{~cm}^{-1}$. This phenomenon maybe a consequence of the presence of the polymer chains at the surface which overlap with the small Raman peaks, and also to heterogeneous broadening.

The average luminescence/Raman spectrum of control composite NPs absent in SiQDs (Fig. 3c) shows a significant SERS effect as evidenced from the four-fold increase in intensity of the most intense band (aromatic stretching band) in comparison to the PS-SERS-NPs. A five-fold increase in SERS intensity was observed for the composite NPs relative to un-encapsulated PS-SERS-NPs (Fig. 3d). Furthermore, a broad signal around 1900-7000 $\mathrm{cm}^{-1}$ attributed to the luminescence peak of SiQDs indicates significant luminescence enhancement of $\sim 17$ fold was obtained when compared to polymeric NPs which encapsulate SiQDs only (prepared as described in ref. 12). Presumably this enhancement arises because the SiQDs are in close proximity to PS-SERS-NPs within the polymer NPs, allowing the intense field of the Au-Ag alloy NPs surface to couple strongly to the SiQDs. It is difficult to directly compare the SERS enhancement obtained in this study with those reported in the literature ${ }^{12}$ because the degree of NP aggregation and the concentration of SERS reporter molecules are often not reported. However, the resulting luminescence enhancement of the composite $\mathrm{NP}$ correlates with $\mathrm{Au}-\mathrm{Si}$ polymer composite NPs reported by us previously ${ }^{5}$ and a rough estimate of the SERS enhancement, taking account of the difference in number of molecules in the focal volume between the 2-naphthalenethiol film and in the composite PS-SERSNPs, gives a value of order $10^{3}$. It is worth noting that, unlike in the case of pure rough silver electrodes, where massive SERS enhancements up to a factor of $10^{6}$ can be achieved, this study involved a composite whose main component is a SERS inactive polymer and therefore the enhancement appears small only because fewer 2-napthalenethiol molecules are present in the composite than in a film of the pure SERS-active component. Nevertheless, the enhanced intensity of SERS bands, together with the broad, enhanced luminescence enhancement signals of SiQDs, indicates that the co-encapsulation of PS-SERS-NPS leads to a significant and simultaneous increase in both SERS and luminescence characteristics.

An approach to enhance both SERS and luminescence simultaneously is reported in which $\mathrm{Au}-\mathrm{Ag}$ alloy NPs encoded with Raman reporter molecules and SiQDs are co-encapsulated inside polymer nanoparticles using miniemulsion polymerization techniques. Simultaneous enhancements in the luminescence of SiQDs ( $\sim 17$ fold compared to the absence of Au-Ag NPs) and SERS (up to five-fold compared to films of the reporter) were observed. It should be emphasized that the simultaneous SERSluminescence enhancement is possible only because of the large Stokes shift of SiQDs. These multifunctional polymer composite nanoparticles possess the potential to be utilized as SERS-based probes in numerous applications, especially in biological labelling and anti-counterfeiting.

We thank the Ministry of Higher Education, Malaysia and Universiti Malaysia Terengganu for generous support and providing graduate funding. We also would like to acknowledge Prof. Robin Harris and the Electron Microscopy Research Service, Newcastle University for the help acquiring TEM images.

\section{Notes and references}

1 N. O'Farrell, A. Houlton and B. R. Horrocks, Int. J. Nanomed., 2006, 1, 451-472.

2 A. Franceschetti and S. T. Pantelides, Phys. Rev. B: Condens. Matter Mater. Phys., 2003, 68, 033313.

3 (a) I. A. Larmour and D. Graham, Analyst, 2011, 136, 3831-3853; (b) G. McNay, D. Eustace, W. E. Smith, K. Faulds and D. Graham, Appl. Spectrosc., 2011, 65, 825-837.

4 (a) S. Biteen, N. S. Lewis, H. A. Atwater, H. Mertens and A. Polman, Appl. Phys. Lett., 2006, 88, 131109; (b) J. S. Biteen, D. Pacifici, N. S. Lewis and H. A. Atwater, Nano Lett., 2005, 5, 1768-1773.

5 N. A. Harun, M. J. Benning, B. R. Horrocks and D. A. Fulton, Nanoscale, 2013, 5, 3817-3827.

$6 \mathrm{Au}-\mathrm{Ag}$ NPs were prepared following the procedure reported by El-Sayed and coworkers: S. Link, Z. L. Wang and M. A. El-Sayed, J. Phys. Chem. B, 1999, 103, 3529-3533.

7 K. Y. van Berkel and C. J. Hawker, J. Polym. Sci., Part A: Polym. Chem., 2010, 48, 1594-1606.

8 Z. Merican, T. L. Schiller, C. J. Hawker, P. M. Fredericks and I. Blakey, Langmuir, 2007, 23, 10539-10545.

9 (a) N. Joumaa, P. Toussay, M. Lansalot and A. Elaissari, J. Polym. Sci., Part A: Polym. Chem., 2008, 46, 327-340; (b) F. Tiarks, K. Landfester and M. Antonietti, Macromol. Chem. Phys., 2001, 202, 51-60.

10 S. Fateixa, A. V. Girao, H. I. S. Nogueira and T. Trindade, J. Mater. Chem., 2011, 21, 15629-15636.

11 (a) R. A. Alvarez-Puebla, D. S. Dos Santos Jr and R. F. Aroca, Analyst, 2004, 129, 1251-1256; (b) F. René, Rep. Prog. Phys., 1998, 61, 691.

12 (a) S. Fateixa, P. C. Pinheiro, H. I. S. Nogueira and T. Trindade, Spectrochim. Acta, Part A, 2013, 113, 100-106; (b) W. Lin, Appl. Phys. A: Mater. Sci. Process., 2011, 102, 121-125. 